\title{
Quenching of acoustic bandgaps by flow noise
}

\author{
T. Elnady, ${ }^{1}$ A. Elsabbagh, ${ }^{1}$ W. Akl, ${ }^{1}$ O. Mohamady, ${ }^{1}$ V. M. Garcia-Chocano, ${ }^{2}$ D. Torrent,${ }^{2}$ \\ F. Cervera, ${ }^{2}$ and J. Sánchez-Dehesa, a) \\ ${ }^{1}$ ASU Sound and Vibration Laboratory, Faculty of Engineering, Ain Shams University 11517 Cairo, Egypt \\ ${ }^{2}$ Wave Phenomena Group, Department of Electronic Engineering, Polytechnic University of Valencia, \\ C/Camino de Vera s.n., E-46022 Valencia, Spain
}

(Received 10 December 2008; accepted 10 March 2009; published online 1 April 2009)

\begin{abstract}
We report an experimental study of acoustic effects produced by wind impinging on noise barriers based on two-dimensional sonic crystals with square symmetry. We found that the attenuation strength of sonic-crystal bandgaps decreases for increasing values of flow speed. A quenching of the acoustic bandgap appears at a certain speed value that depends of the barrier filling ratio. For increasing values of flow speed, the data indicate that the barrier becomes a sound source because of its interaction with the wind. We conclude that flow noise should be taken into account in designing acoustic barriers based on sonic crystals. (C) 2009 American Institute of Physics.
\end{abstract}

[DOI: $10.1063 / 1.3111797$ ]

A sonic crystal (SC) is a periodic array of sound scatterers in air. SCs based on two-dimensional (2D) distributions of cylindrical scatterers have been mainly studied in view of their possible applications as acoustic barriers. ${ }^{1-9}$ Hexagonal and square configurations of cylinders are those widely studied, but other combinations made of them have been also analyzed. ${ }^{3,4}$ For the case in which the scatterers are made by solid materials, such as aluminum or concrete, the physical mechanism giving the transmission loss through the barrier is controlled by Bragg reflection between successive planes of scatterers in the barrier. The phenomenon of Bragg reflection creates frequency bands (bandgaps) at which the sound propagation is forbidden. Cylindrical scatterers with circular section are mainly studied although sections like square, hexagonal, and many others have been also considered looking for stronger and wider complete bandgaps. ${ }^{8,9}$ More recently, additional mechanisms of sound reduction have been essayed in order to improve the barrier's performance in the range of frequencies not covered by the bandgaps. For example, the absorption mechanism associated to a porous covering of the scatterers would produce additional sound reduction in a wide range of frequencies. ${ }^{10}$ Another possibility is using periodic arrays of trees, which also produce a reduction in the transmitted sound by natural means. ${ }^{11}$ Sound attenuation by outdoor barriers based on 2D SCs have already been demonstrated at realistic size. ${ }^{6}$

In this letter we report an experimental study showing that wind impinging on a noise barrier based on a 2D SC produces effects changing its attenuation performance. We analyze here the quenching of SC bandgaps by flow noise, a problem that has not been previously tackled, although, as it is shown below, it has a paramount importance. It is demonstrated that flow noise can completely deteriorate the barrier performance if the speed flow is large enough. We also demonstrate that for barriers too dense (i.e., when the distance between cylinders is too short) the barrier even becomes in a source of noise for large flow speeds.

\footnotetext{
${ }^{\text {a) }}$ Author to whom correspondence should be addressed. Electronic mail: jsdehesa@upvnet.upv.es.
}

In order to quantify the attenuation performance of SC barriers under the effect of impinging wind, a series of measurements are developed in a wind tunnel consisting of a duct $6 \mathrm{~m}$ long that has been constructed in the ASU laboratory. The duct is constructed to guide the air flow and has a section of $29.2 \mathrm{~cm}$ (wide) $\times 31.2 \mathrm{~cm}$ (high) for free air passage. The SC samples are placed in the first half of the duct by removing a part specially designed on the bottom wall.

Figure 1 shows a schematic view of the experimental setup. Two loudspeakers are mounted at the entrance to generate sound inside the duct. A Pitot tube with a built in thermocouple is placed in front of the sample to measure the incident flow. All reported flows are measured at the duct center. Two BSWA MPA-201 half-inch microphones are mounted at about 0.5 and $1 \mathrm{~m}$ upstream and downstream the $\mathrm{SC}$, respectively. The upstream microphone (Mic1) records the pressure level before its arrival to the sample and it is used to ensure that we provide the same excitation (i.e., the same incident sound wave) for all the samples. The downstream microphone (Mic2) records the sound pressure level (SPL) after passing through the SC samples and it is used to calculate the insertion loss (IL) for each SC sample. The data is collected with a National Instruments Data Acquisition Systems consisting of a PXI chassis equipped with a PXI4472 Data Acquisition Card for microphone input, PXI-6733 for loudspeaker output, and PXI-6221 for reading the digital manometer, the flow temperature, and controlling the fan speed through a frequency inverter. The fan maximum rating $5 \mathrm{~m} / \mathrm{s}$ at $5 \mathrm{kPa}$. Data are recorded up to $20 \mathrm{kHz}$. Here, we analyze and plot results only up to $8 \mathrm{kHz}$ since the first

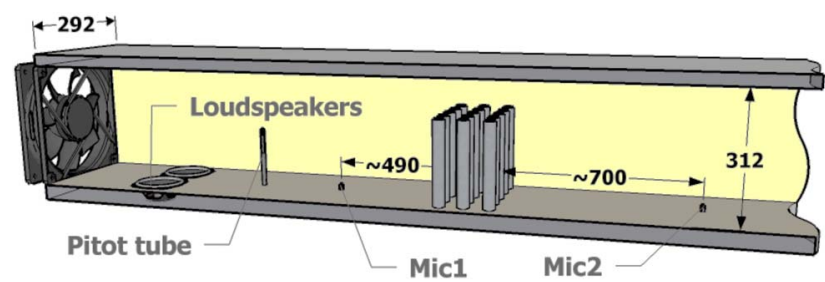

FIG. 1. (Color online) Scheme of the experimental setup inside the duct. The distances are in millimeter. 
TABLE I. Diameter of cylinders $d$ (in millimeter) employed in the construction of acoustic barriers. For each $d$, three different samples are built by using SCs with square symmetry and lattice parameter $a$ (in millimeter). The corresponding filling ratio $f$ (in percent) is given between square parenthesis.

\begin{tabular}{cccc}
\hline \hline$d$ & \multicolumn{3}{c}{$a$} \\
\hline 16.7 & $19(61 \%)$ & $30(24 \%)$ & $38(15 \%)$ \\
24.6 & $30(53 \%)$ & $40(30 \%)$ & $50(19 \%)$ \\
31.8 & $39(52 \%)$ & $49(33 \%)$ & $60(22 \%)$ \\
\hline \hline
\end{tabular}

acoustic bandgap of samples under study appears in this frequency range.

The SC samples are constructed by using hollow metal cylinders $25 \mathrm{~cm}$ long, and thick enough so that they are acoustically rigid. Each sample is made up of only three rows of cylinders put in a square configuration. We used three rows because, as it is explained below, our simulations demonstrate that three rows are enough to get robust acoustic bandgaps. Cylinders with three different diameter size $d$ are employed. For each size, three different lattice parameter $a$ are used to build the corresponding SC samples. The geometrical parameters of the nine different samples analyzed are reported in Table I, which also gives the filling ratio $f$ (in percentage) of the corresponding square lattice; i.e, $f=\pi(d / 2 a)^{2}$. Note that the condition of maximum filling ratio (close packing condition) is achieved for $f \approx 0.79 \%$.

The IL for a given SC sample is calculated by subtracting the SPL measured with Mic2 from a reference, which is the SPL measured when no sample is inside the duct

$$
\mathrm{IL}=\mathrm{SPL}_{\mathrm{ref}}-\mathrm{SPL}_{\mathrm{SC}} \text {. }
$$

A positive IL means that the sound is attenuated while a negative IL means that the sound increases.

Two sets of measurements are performed. For the first set we have recorded the flow noise when the speakers are turned off (no sound excitation), and the fan is running at different speeds delivering $v=5,10,15$, and $20 \mathrm{~m} / \mathrm{s}$. Figure 2 shows the IL measured for the samples constructed by using $24.6 \mathrm{~mm}$ cylinders (middle row in Table I). Note that for all the $f$ values analyzed the spectra show a broad deep that is centered at about $2.3 \mathrm{kHz}$ for the case $5 \mathrm{~m} / \mathrm{s}$ flow speed. It represents an aerodynamic noise ${ }^{12}$ since, on the one hand, the center frequency shifts upwards with increasing flow speed (see Fig. 2). On the second hand, a comparison with data taken from samples made of cylinders with different diameter (i.e., 16.7 and $31.8 \mathrm{~mm}$ ) indicates that the broad noise shifts to lower (larger) frequencies for larger (smaller) $d$. For the larger filling ratio (53\%) the flow noise generated increases by almost $10 \mathrm{~dB}$ and it is accompanied by an interesting phenomenon associated to the periodicity of the structure. Note how additional deeps appear, which do not shift with the flow speed, and they are located at frequencies of maximum transmittance (zero reflectance) by the structure. This claim is supported by the calculated reflectance depicted on the top panel in Fig. 2. Note that for all the flow speeds the narrow noise centered at about $2.8 \mathrm{kHz}$ corresponds to a zero on the reflectance spectrum. Moreover, the shoulder observed at about $8.5 \mathrm{kHz}$ on the IL spectrum corresponding to the flow speed of $20 \mathrm{~m} / \mathrm{s}$ is associated to the zero obtained for the reflectance spectrum. The shoulder (much weaker) also appear on the IL spectrum for $15 \mathrm{~m} / \mathrm{s}$ but
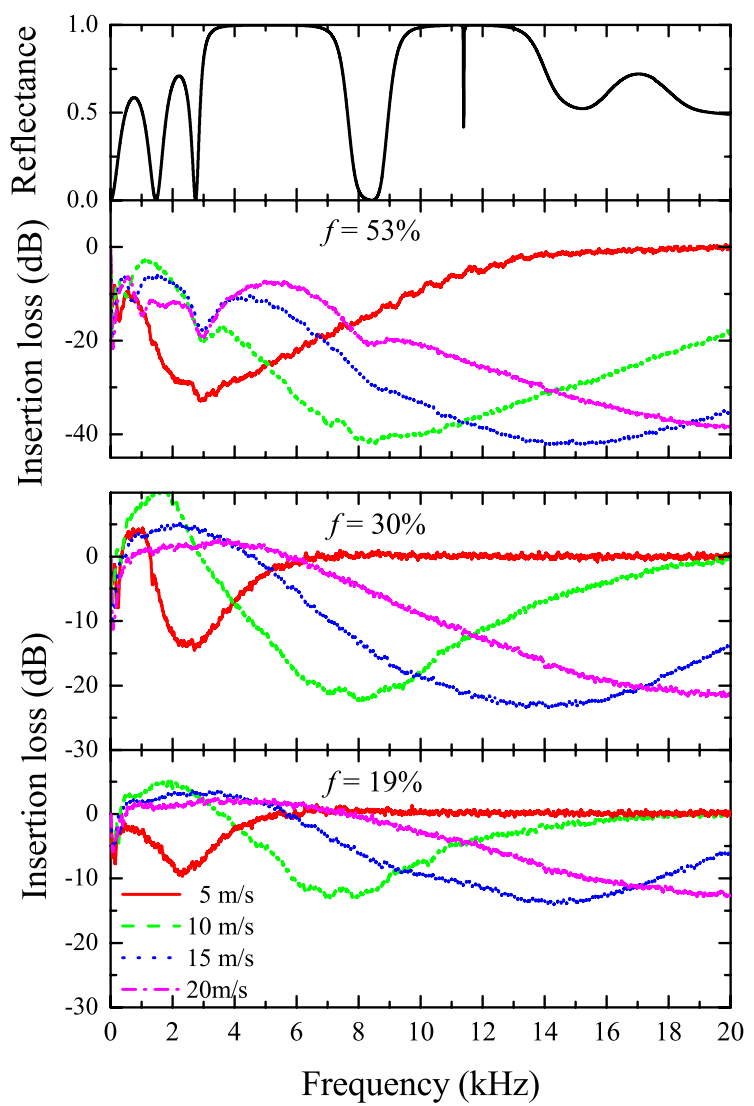

FIG. 2. (Color online) IL (in decibel) as a function of the frequency when there is no sound excitation in the duct. The three samples analyzed consist of three rows of $24.6 \mathrm{~mm}$, cylinder's diameter, put in a square configuration and have different filling ratio $f$ (see Table I). The top panel represents the reflectance calculated for the structure with $f=0.53$.

is not observed on the other two spectra. These features indicate that the flow noise generated on the first row of cylinders crosses the structure and experiments the Bragg scattering by the following rows of cylinders. This phenomenon is extremely interesting since it opens the possibility of tailoring the flow noise generated by this type of ordered structures. A deep analysis of this phenomenon is out of the scope of the present short article.

The second set of experiment corresponds to the case when the sound is excited inside the duct (loudspeakers on) and it is applied together with the wind flow. Figure 3 represents the IL spectra recorded for the samples made by using the cylinders of $31.8 \mathrm{~mm}$. Each color line defines the spectrum associated to one flow speed, the black line being the case of no wind flow. This figure also depicts the reflectance spectra corresponding to each filling ration, which has been calculated by a multiple scattering algorithm previously developed. ${ }^{5,13}$ Let us remark that the reflectance has been calculated with the approximation of infinitely long cylinders. For the case of no flow the IL profiles qualitatively agree with that of the calculated reflectance. Particularly, the first acoustic bandgap, as it is determined by reflectance simulations, fairly agrees with the broad peak observed at any IL spectra with no flow. This result supports our assumption that only three rows of scatterers are enough to get a robust acoustic bandgap.

Figure 3 also shows that the area under the IL peak associated to the bandgap decreases with increasing flow speed. We have considered this area as a parameter measur- 


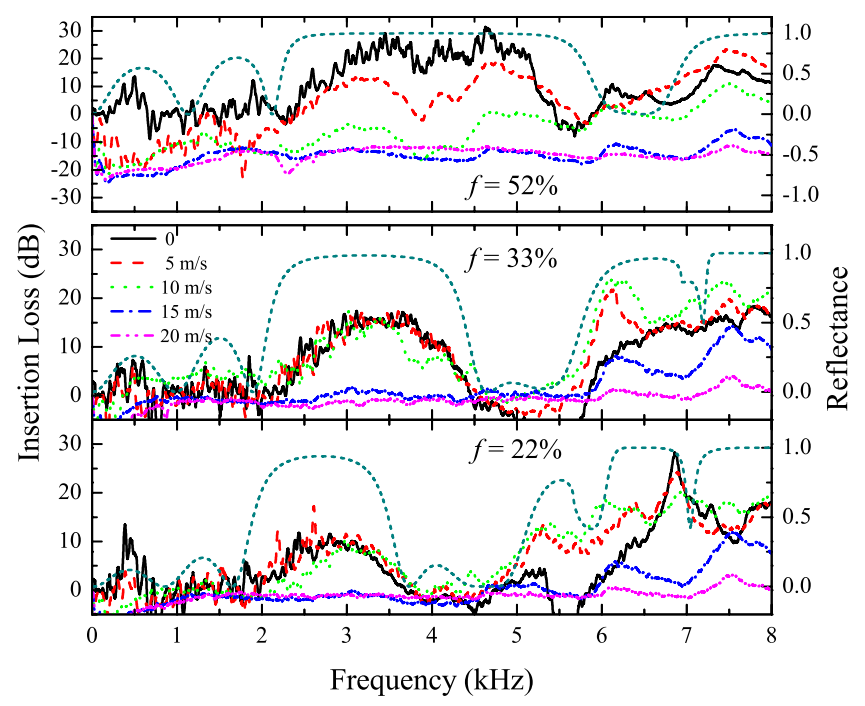

FIG. 3. (Color online) IL (in decibel) of acoustic barrier made of three rows of rigid cylinders (with diameter of $31.8 \mathrm{~mm}$ ) put in a square configuration with three different filling ratios. The black curves correspond to the case of no flow impinging over the barrier. The color lines define the four flow speeds analyzed. The dashed lines represent the reflectance calculated by a multiple scattering method and their values are defined by the right scale.

ing the attenuation strength of the barrier at the bandgap and its behavior, as a function of flow speed, is plotted in Fig. 4 for all the samples in Table I. It is concluded that the attenuation strength decreases with increasing flow speed. A quenching of the acoustic bandgap (zero area under the IL peak) appears for a certain value of flow speed that depends on the SC's filling ratio; this cutoff value is larger for lower filling ratios. More interesting, it is the remarkable effect observed for dense barriers $(\geq 53 \%)$. Note that after the

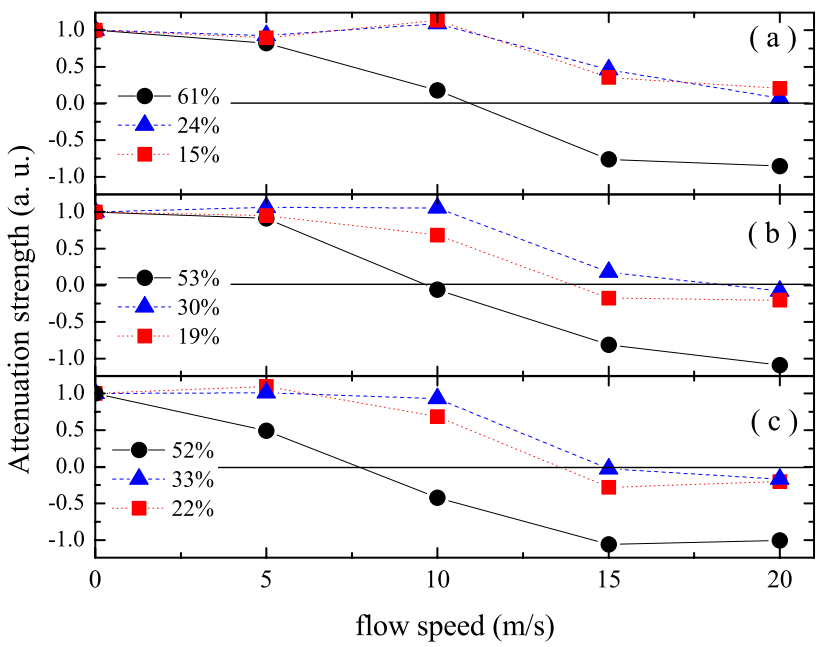

FIG. 4. (Color online) Attenuation strength (see text) at bandgap frequencies as a function of flow speed for SC samples based on cylinders of diameter (a) $16.7 \mathrm{~mm}$, (b) $24.6 \mathrm{~mm}$, and (c) $31.8 \mathrm{~mm}$. See Table I for more details. A negative value means that the SC generates noise at the bandgap frequencies. bandgap quenching, the barrier becomes a sound source in the same frequency region (i.e., the peak in the IL spectrum becomes in a deep) due to flow noise. This behavior is physically understood by the vortex formation (generating noise) caused by air flow passing trough the small apertures between cylinders. For moderate and low dense barrier $(f$ $\leq 30 \%$ ), where the bandgap quenching appears at much larger flow speed, the attenuation effect at bandgap frequencies disappear after bandgap quenching and the barrier becomes transparent to sound. However, it is reasonable to expect intermediate results for samples with $f$ values between $30 \%$ and $52 \%$. These measurements in a duct are of extraordinary importance and should be taken into account in designing outdoor attenuation barriers based on SCs.

In summary, we have demonstrated that the attenuation effects of noise barriers based on SC can be completely destroyed by impinging air flow. Moreover, the barrier even becomes a noisy source for large enough flow speed. Therefore, flow noise is a relevant phenomenon in controlling the attenuation properties of SC's acoustic barriers working in windy environments. We hope that our results stimulate further theoretical work and new measurements by using actual size samples in open air.

This work was supported by the Spanish Agency of International Cooperation (AECI), the Spanish Ministry of Science and Innovation (MICIIN), and the Spanish Ministry of Public Works (MF). J.S.-D. acknowledges useful conversations with A. Broatch.

${ }^{1}$ J. V. Sánchez-Perez, D. Caballero, R. Martínez-Sala, C. Rubio, J. SánchezDehesa, F. Meseguer, J. Llinares, and F. Gálvez, Phys. Rev. Lett. 80, 5325 (1998).

${ }^{2}$ C. Rubio, D. Caballero, J. V. Sánchez-Pérez, R. Martínez-Sala, J. SánchezDehesa, F. Meseguer, and F. Cervera, J. Lightwave Technol. 17, 2202 (1999).

${ }^{3}$ D. Caballero, J. Sánchez-Dehesa, C. Rubio, R. Martínez-Sala, J. V. Sánchez-Pérez, F. Meseguer, and J. Llinares, Phys. Rev. E 60, R6316 (1999).

${ }^{4}$ D. Caballero, J. Sánchez-Dehesa, R. Martínez-Sala, C. Rubio, J. V. Sánchez-Pérez, L. Sanchis, and F. Meseguer, Phys. Rev. B 64, 064303 (2001).

${ }^{5}$ Y.-Y. Chen and Z. Ye, Phys. Rev. Lett. 87, 184301 (2001).

${ }^{6}$ J. V. Sánchez-Perez, C. Rubio, R. Martínez-Sala, R. Sánchez-Grandia, and V. Gomez, Appl. Phys. Lett. 81, 5240 (2002).

${ }^{7}$ C. Goffaux, F. Maseri, J. O. Vasseur, D. Djafari-Rouhani, and Ph. Lambin, Appl. Phys. Lett. 83, 281 (2003).

${ }^{8}$ C. Goffaux and J. P. Vigneron, Phys. Rev. B 64, 075118 (2001).

${ }^{9}$ L. Zhong, F. Wu, X. Zhang, H. Zhong, and S. Zhong, Phys. Lett. A 339, 164 (2005).

${ }^{10}$ O. Umnova, K. Attenborough, and C. M. Linton, J. Acoust. Soc. Am. 119, 278 (2006).

${ }^{11}$ R. Martínez-Sala, C. Rubio, L.M. Garcia-Raffi, J.V. Sánchez-Perez, E.A. Sánchez-Perez, and J. Llinares, J. Sound Vib. 291, 100 (2006).

${ }^{12}$ M. S. Howe and H. D. Baumann, in Noise and Vibration Control Engineering, edited by L. L. Beranek and I. L. Ver (Wiley, New York, 1992), Chap. 14.

${ }^{13}$ L. Sanchis, A. Håkansson, F. Cervera, and J. Sánchez-Dehesa, Phys. Rev. B 67, 035422 (2003). 